\title{
A locus on 19p13 modifies risk of breast cancer in BRCA1 mutation carriers and is associated with hormone receptor- negative breast cancer in the general population
}

Antonis C Antoniou ${ }^{1}$, Xianshu Wang ${ }^{2}$, Zachary S Fredericksen ${ }^{3}$, Lesley McGuffog ${ }^{1}$, Robert Tarrell $^{3}$, Olga M Sinilnikova ${ }^{4,5}$, Sue Healey ${ }^{6}$, Jonathan Morrison ${ }^{1}$, Christiana Kartsonaki ${ }^{1}$, Timothy Lesnick ${ }^{3}$, Maya Ghoussaini ${ }^{6}$, Daniel Barrowdale ${ }^{1}$, EMBRACE ${ }^{1,133}$, Susan Peock ${ }^{1}$, Margaret Cook $^{1}$, Clare Oliver $^{1}$, Debra Frost ${ }^{1}$, Diana Eccles ${ }^{7}$, D Gareth Evans ${ }^{8}$, Ros Eeles ${ }^{9}$, Louise Izatt ${ }^{10}$, Carol Chu ${ }^{11}$, Fiona Douglas ${ }^{12}$, Joan Paterson ${ }^{13}$, Dominique StoppaLyonnet $^{14}$, Claude Houdayer ${ }^{14}$, Sylvie Mazoyer ${ }^{5}$, Sophie Giraud ${ }^{4}$, Christine Lasset ${ }^{15,16}$, Audrey Remenieras ${ }^{17}$, Olivier Caron ${ }^{18}$, Agnès Hardouin ${ }^{19}$, Pascaline Berthet $^{19}$, GEMO Study Collaborators ${ }^{20,133}$, Frans B L Hogervorst ${ }^{21}$, Matti A Rookus ${ }^{22}$, Agnes Jager ${ }^{23}$, Ans van den Ouweland ${ }^{24}$, Nicoline Hoogerbrugge ${ }^{25}$, Rob B van der Luijt ${ }^{26}$, Hanne MeijersHeijboer $^{27}$, Encarna B Gómez García ${ }^{28}$, HEBON ${ }^{29,133}$, Peter Devilee ${ }^{30,31}$, Maaike P G Vreeswijk $^{32}$, Jan Lubinski ${ }^{33}$, Anna Jakubowska ${ }^{33}$, Jacek Gronwald ${ }^{33}$, Tomasz Huzarski ${ }^{33}$, Tomasz Byrski ${ }^{33}$, Bohdan Górski ${ }^{33}$, Cezary Cybulski ${ }^{33}$, Amanda B Spurdle ${ }^{6}$, Helene Holland $^{6}$, kConFab ${ }^{34,133}$, David E Goldgar ${ }^{35}$, Esther M John ${ }^{36}$, John L Hopper ${ }^{37}$, Melissa Southey $^{37}$, Saundra S Buys ${ }^{38}$, Mary B Daly ${ }^{39}$, Mary-Beth Terry ${ }^{40}$, Rita K Schmutzler ${ }^{41,42}$, Barbara Wappenschmidt ${ }^{41,42}$, Christoph Engel ${ }^{43}$, Alfons Meindl ${ }^{44}$, Sabine PreislerAdams $^{45}$, Norbert Arnold ${ }^{46}$, Dieter Niederacher ${ }^{47}$, Christian Sutter ${ }^{48}$, Susan M Domchek ${ }^{49}$, Katherine L Nathanson ${ }^{49}$, Timothy Rebbeck ${ }^{49}$, Joanne L Blum ${ }^{50}$, Marion Piedmonte ${ }^{51}$, Gustavo C Rodriguez ${ }^{52}$, Katie Wakeley ${ }^{53}$, John F Boggess ${ }^{54}$, Jack Basil ${ }^{55}$, Stephanie V

\footnotetext{
(C) 2010 Nature America, Inc. All rights reserved.

Correspondence should be addressed to F.J.C. (couch.fergus@mayo.edu).

$133 \mathrm{~A}$ full list of members is provided in the supplementary Note.

Note: Supplementary information is available on the Nature Genetics website.

AUTHOR CONTRIBUTIONS

F.J.C., A.C.A. and D.F.E. designed the study and obtained financial support. G.C.-T. founded CIMBA in order to provide the infrastructure for the BRCA1 GWAS. F.J.C. and X.W. coordinated collection of samples. A.C.A. directed the statistical analysis D.F.E. advised on the statistical analysis. C.K., Z.S.F. and T.L. carried out analyses. Z.S.F., R.T., J.M., L.M. and D.B. provided bioinformatics and database support. F.J.C., H. Hakonarson and X.W. directed the genotyping of the BRCAl carrier and triplenegative samples. M.G. directed the genotyping of the UK case-control samples. A.C.A., F.J.C. and D.F.E. drafted the manuscript. F.J.C. was the overall project leader.

O.M.S. and S.H. coordinated the BRCA1 mutation classification. T.K., J.V., M.M.G., D.A. and C.G. were involved in the BRCA2 GWAS genotyping and coordination. K.O. led the BRCA2 GWAS.

S.P., M.C., C.O., D.F., D.E., D.G.E., R.E., L.I., C.C., F.D., J.P., O.M.S., D.S.-L., C.H., S.M., S.G., C.L., A.R., O.C., A.H., P.B., F.B.L.H., M.A.R., A.J., A.v.d.O., N.H., R.B.v.d.L., H.M.-H., E.B.G.G., P.D., M.P.G.V., J.L., A.J., J.G., T.H., T.B., B.G., C.C., A.B.S., H.H., D.E., E.M.J., J.L.H., M.S., S.S.B., M.B.D., M.-B.T., R.K.S., B.W., C.E., A.M., S.P.-A., N.A., D.N., C.S., S.M.D., K.L.N., T.R., J.L.B., M.P., G.C.R., K.W., J.F.B., J.B., S.V.B., E.F., B.K., Y.L., R.M., I.L.A., G.G., H.O., N.L., K.H., J.R., H.E., A.M.G., M.T., L.S., P.P., S.M., B.B., A.V., P.R., T.C., M.d.1.H., C.F.S., A.F.-R., M.H.G., P.L.M., J.T.L., L.G., N.M.L., T.V.O.H., F.C.N., I.B., C.L., J.G., S.J.R., S.A.G., C.P., S.N., C.I.S., J.B., A.O., H.N., T.H., M.A.C., M.S.B., U.H., A.K.G., M.M., C.C., S.L.N., B.Y.K., N.T., A.E.T., J.W., O.O., J.S., P.S., W.S.R., A.A. and G.R. collected data and samples on $B R C A 1$ and or BRCA2 mutation carriers.

N.G.M., G.W.M., J.C.-C., D.F.-J., H.B., G.S., L.B., A.C., S.S.C., P.M., S.M.G., W.T., D.Y., G.F., P.A.F., M.W.B., I.d.S.S., J.P., D.L., R.P., T.R., A.F., R.W., K.P., R.B.D., A.M.L., J.E.-P., C.V., F.B., K.D., A.D. and P.P.D.P. collected data and samples for the TNBCC case-control and/or the SEARCH studies.
}

All authors provided critical review of the manuscript.

COMPETING FINANCIAL INTERESTS

The authors declare no competing financial interests.

Reprints and permissions information is available online at http://npg.nature.com/reprintsandpermissions/. 
Blank $^{56}$, Eitan Friedman ${ }^{57}$, Bella Kaufman ${ }^{58}$, Yael Laitman ${ }^{57}$, Roni Milgrom ${ }^{57}$, Irene L Andrulis $^{59,60,61}$, Gord Glendon ${ }^{59}$, Hilmi Ozcelik ${ }^{60}$, Tomas Kirchhoff 62,63 , Joseph Vijai62,63, Mia M Gaudet ${ }^{64,65}$, David Altshuler ${ }^{66}$, Candace Guiducci ${ }^{66}$, SWE-BRCA ${ }^{67,133}$, Niklas Loman $^{68}$, Katja Harbst ${ }^{68}$, Johanna Rantala ${ }^{69}$, Hans Ehrencrona ${ }^{70}$, Anne-Marie Gerdes ${ }^{71}$, Mads Thomassen ${ }^{72}$, Lone Sunde ${ }^{73}$, Paolo Peterlongo ${ }^{74,75}$, Siranoush Manoukian ${ }^{76}$, Bernardo Bonanni ${ }^{77}$, Alessandra Viel ${ }^{78}$, Paolo Radice ${ }^{74,75}$, Trinidad Caldes $^{79}$, Miguel de la Hoya $^{77}$, Christian F Singer ${ }^{80}$, Anneliese Fink-Retter ${ }^{80}$, Mark H Greene ${ }^{81}$, Phuong L Mai ${ }^{81}$, Jennifer T Loud ${ }^{81}$, Lucia Guidugli ${ }^{2}$, Noralane M Lindor ${ }^{82}$, Thomas V O Hansen ${ }^{83}$, Finn C Nielsen ${ }^{83}$, Ignacio Blanco ${ }^{84}$, Conxi Lazaro ${ }^{84}$, Judy Garber ${ }^{85}$, Susan J Ramus ${ }^{86}$, Simon A Gayther $^{86}$, Catherine Phelan ${ }^{87}$, Stephen Narod ${ }^{88}$, Csilla I Szabo², MOD SQUAD ${ }^{89,133}$, Javier Benitez $^{90}$, Ana Osorio ${ }^{90}$, Heli Nevanlinna ${ }^{91}$, Tuomas Heikkinen ${ }^{91}$, Maria A Caligo ${ }^{92}$, Mary S Beattie $^{93,94,95}$, Ute Hamann ${ }^{96}$, Andrew K Godwin ${ }^{39}$, Marco Montagna ${ }^{97}$, Cinzia Casella ${ }^{97}$, Susan L Neuhausen ${ }^{98}$, Beth Y Karlan ${ }^{99,100}$, Nadine Tung ${ }^{101}$, Amanda E Toland ${ }^{102}$, Jeffrey Weitzel $^{103}$, Olofunmilayo Olopade ${ }^{104}$, Jacques Simard ${ }^{105,106}$, Penny Soucy ${ }^{105}$, Wendy S Rubinstein $^{107}$, Adalgeir Arason ${ }^{108}$, Gad Rennert ${ }^{109}$, Nicholas G Martin ${ }^{110}$, Grant W Montgomery ${ }^{110}$, Jenny Chang-Claude ${ }^{111}$, Dieter Flesch-Janys ${ }^{112}$, Hiltrud Brauch ${ }^{113,114}$, GENICA $^{115,133}$, Gianluca Severi ${ }^{116}$, Laura Baglietto ${ }^{116}$, Angela Cox ${ }^{117}$, Simon S Cross ${ }^{118}$, Penelope Miron ${ }^{119}$, Sue M Gerty ${ }^{7}$, William Tapper ${ }^{7}$, Drakoulis Yannoukakos ${ }^{120}$, George Fountzilas $^{121,122}$, Peter A Fasching ${ }^{123}$, Matthias W Beckmann ${ }^{124}$, Isabel dos Santos Silva ${ }^{125}$, Julian Peto ${ }^{125}$, Diether Lambrechts ${ }^{126}$, Robert Paridaens ${ }^{127}$, Thomas Rüdiger ${ }^{128}$, Asta Försti ${ }^{96,129}$, Robert Winqvist ${ }^{130}$, Katri Pylkäs ${ }^{130}$, Robert B Diasio ${ }^{131}$, Adam M Lee ${ }^{131}$, Jeanette Eckel-Passow ${ }^{3}$, Celine Vachon ${ }^{3}$, Fiona Blows ${ }^{1}$, Kristy Driver ${ }^{1}$, Alison Dunning ${ }^{1}$, Paul P D Pharoah ${ }^{1}$, Kenneth Offit ${ }^{61}$, V Shane Pankratz ${ }^{3}$, Hakon Hakonarson ${ }^{132}$, Georgia Chenevix-Trench ${ }^{6}$, Douglas F Easton ${ }^{1}$, and Fergus $\mathrm{J} \mathrm{Couch}^{2}$

${ }^{1}$ Centre for Cancer Genetic Epidemiology, Department of Public Health and Primary Care, University of Cambridge, Cambridge, UK ${ }^{2}$ Department of Laboratory Medicine and Pathology, Mayo Clinic, Rochester, Minnesota, USA ${ }^{3}$ Department of Health Sciences Research, Mayo Clinic, Rochester, Minnesota, USA ${ }^{4}$ Unité Mixte de Génétique Constitutionnelle des Cancers Fréquents, Centre Hospitalier Universitaire de Lyon/Centre Léon Bérard, Lyon, France ${ }^{5}$ Equipe labellisée LIGUE 2008, UMR5201 CNRS, Centre Léon Bérard, Université de Lyon, Lyon, France ${ }^{6}$ Queensland Institute of Medical Research, Brisbane, Australia ${ }^{7}$ Wessex Clinical Genetics Service, Princess Anne Hospital, Southampton, UK ${ }^{8}$ Genetic Medicine, Manchester Academic Health Sciences Centre, Central Manchester University Hospitals National Health Service (NHSFT) Foundation Trust, Manchester, UK ${ }^{9}$ Oncogenetics Team, The Institute of Cancer Research and Royal Marsden NHS Foundation Trust, Sutton, UK ${ }^{10}$ Clinical Genetics, Guy's and St. Thomas' NHS Foundation Trust, London, UK ${ }^{11}$ Yorkshire Regional Genetics Service, Leeds, UK ${ }^{12}$ Institute of Human Genetics, Centre for Life, Newcastle Upon Tyne Hospitals NHS Trust, Newcastle upon Tyne, UK ${ }^{13}$ Department of Clinical Genetics, East Anglian Regional Genetics Service, Addenbrookes Hospital, Cambridge, UK ${ }^{14}$ INSERM U509, Service de Génétique Oncologique, Institut Curie, Université Paris-Descartes, Paris, France ${ }^{15}$ CNRS UMR5558, Université Lyon 1, Lyon, France ${ }^{16}$ Unité de Prévention et d'Epidémiologie Génétique, Centre Léon Bérard, Lyon, France ${ }^{17}$ Genetics Department, Institut de Cancérologie Gustave Roussy, Villejuif, France ${ }^{18}$ Consultation de Génétique, Département de Médecine, Institut de Cancérologie Gustave Roussy, Villejuif, France ${ }^{19}$ Centre François Baclesse, Caen, France 20 GEMO study, Cancer Genetics Network 'Groupe Génétique et Cancer', Fédération Nationale des Centres de Lutte Contre le Cancer, Paris, France ${ }^{21}$ Family Cancer Clinic, Netherlands Cancer Institute, Amsterdam, The Netherlands 22 Department of Epidemiology, Netherlands Cancer Institute, Amsterdam, The Netherlands ${ }^{23}$ Department of Medical Oncology, Rotterdam Family Cancer Clinic, Erasmus University Medical Center, Rotterdam, The Netherlands ${ }^{24}$ Department of Clinical Genetics, Rotterdam Family Cancer Clinic, Erasmus University Medical Center, Rotterdam, The Netherlands ${ }^{25}$ Department of Human Genetics, Radboud University Nijmegen 
Medical Centre, Nijmegen, The Netherlands ${ }^{26}$ Department of Medical Genetics, University Medical Center Utrecht, Utrecht, The Netherlands ${ }^{27}$ Department of Clinical Genetics, Vrije Universiteit (VU) Medical Center, Amsterdam, The Netherlands ${ }^{28}$ Department of Clinical Genetics, School for Oncology and Developmental Biology, Maastricht University Medical Center, Maastricht, The Netherlands ${ }^{29}$ HEBON, Hereditary Breast and Ovarian Cancer Research Group, The Netherlands ${ }^{30}$ Department of Human Genetics, Leiden University Medical Center, Leiden, The Netherlands ${ }^{31}$ Department of Pathology, Leiden University Medical Center, Leiden, The Netherlands ${ }^{32}$ Department of Toxicogenetics, Leiden University Medical Center, Leiden, The Netherlands ${ }^{33}$ International Hereditary Cancer Center, Department of Genetics and Pathology, Pomeranian Medical University, Szczecin, Poland ${ }^{34}$ kConFab, Kathleen Cuningham Foundation Consortium for Research into Familial Breast Cancer, Peter MacCallum Cancer Centre, Melbourne, Australia ${ }^{35}$ Department of Dermatology, University of Utah School of Medicine, Salt Lake City, Utah, USA ${ }^{36}$ Cancer Prevention Institute of California, Stanford University School of Medicine, Stanford, California, USA ${ }^{37}$ The University of Melbourne, Melbourne, Australia ${ }^{38}$ Huntsman Cancer Institute, University of Utah Health Sciences Centre, Salt Lake City, Utah, USA ${ }^{39}$ Fox Chase Cancer Center, Philadelphia, Pennsylvania, USA ${ }^{40}$ Columbia University, New York, New York, USA ${ }^{41}$ Centre of Familial Breast and Ovarian Cancer, Department of Obstetrics and Gynaecology, University of Cologne, Cologne, Germany ${ }^{42}$ Centre for Integrated Oncology (CIO), University of Cologne, Cologne, Germany ${ }^{43}$ Institute for Medical Informatics, Statistics and Epidemiology, University of Leipzig, Leipzig, Germany ${ }^{44}$ Department of Obstetrics and Gynaecology, Division of Tumor Genetics, Klinikum rechts der Isar, Technical University Munich, Munich, Germany ${ }^{45}$ Institute of Human Genetics, University of Muenster, Muenster, Germany ${ }^{46}$ Department of Obstetrics and Gynaecology, University Hospital of Schleswig-Holstein, Campus Kiel, Christian-Albrechts University, Kiel, Germany ${ }^{47}$ Department of Obstetrics and Gynaecology, Division of Molecular Genetics, University Hospital Düsseldorf, Heinrich-Heine University Düsseldorf, Dusseldorf, Germany ${ }^{48}$ Institute of Human Genetics, Division of Molecular Diagnostics, University Heidelberg, Heidelberg, Germany ${ }^{49}$ University of Pennsylvania, Philadelphia, Pennsylvania, USA ${ }^{50}$ Baylor-Charles A. Sammons Cancer Center, Dallas, Texas, USA ${ }^{51}$ Gynecology Oncology Group Statistical and Data Center, Roswell Park Cancer Institute, Buffalo, New York, USA ${ }^{52}$ Northshore University Health System, Evanston Northwestern Healthcare, Evanston, Illinois, USA ${ }^{53}$ Tufts University, New England Medical Center, Boston, Massachusetts, USA ${ }^{54}$ University of North Carolina, Chapel Hill, North Carolina, USA ${ }^{55}$ St. Elizabeth Medical Center, Edgewood, Kentucky, USA ${ }^{56}$ New York University School of Medicine, New York, New York, USA 57 The Susanne Levy Gertner Oncogenetics Unit, Sheba Medical Center, Tel-Hashomer, Israel ${ }^{58}$ Oncology Institute, Sheba Medical Center, Tel-Hashomer, Israel 59 Ontario Cancer Genetics Network, Cancer Care Ontario, Toronto, Ontario, Canada ${ }^{60}$ Fred A. Litwin Center for Cancer Genetics, Samuel Lunenfeld Research Institute, Mount Sinai Hospital, Toronto, Ontario, Canada ${ }^{61}$ Department of Molecular Genetics, University of Toronto, Toronto, Ontario, Canada 62 Clinical Genetics Service, Memorial Hospital, New York, New York, USA 63 Cancer Biology and Genetics Program, Sloan-Kettering Institute, Memorial Sloan-Kettering Cancer Center, New York, New York, USA ${ }^{64}$ Department of Epidemiology and Population Health, Albert Einstein College of Medicine, New York, New York, USA ${ }^{65}$ Department of Obstetrics and Gynecology and Women's Health, Albert Einstein College of Medicine, New York, New York, USA ${ }^{66}$ Broad Institute of Harvard and Massachusetts Institute of Technology (MIT), Cambridge, Massachusetts, USA ${ }^{67}$ SWE-BRCA, Swedish Breast Cancer Study, Sweden 68 Department of Oncology, Lund University, Lund, Sweden ${ }^{69}$ Department of Clinical Genetics, Karolinska University Hospital, Stockholm, Sweden ${ }^{70}$ Department of Genetics and Pathology Rudbeck Laboratory, Uppsala University, Uppsala, Sweden ${ }^{71}$ Department of Clinical Genetics, Rigshospitalet, Copenhagen, Denmark ${ }^{72}$ Department of Clinical Genetics, Odense University Hospital, Odense, Denmark ${ }^{73}$ Department of Clinical Genetics, Aalborg and Aarhus University Hospital, Aaarhus, Denmark ${ }^{74}$ Unit of Genetic Susceptibility to Cancer, Department of 
Experimental Oncology and Molecular Medicine, Fondazione Instituto di Ricovero e Cura a Carattere Scientifico (IRCCS), Istituto Nazionale Tumori (INT), Milan, Italy ${ }^{75}$ IFOM, Fondazione Istituto Fondazione Italiana per la Ricera sul Cancro (FIRC) di Oncologia Molecolare, Milan, Italy 76 Unit of Medical Genetics, Department of Preventive and Predictive Medicine, Fondazione IRCCS Istituto Nazionale dei Tumori (INT), Milan, Italy ${ }^{77}$ Division of Cancer Prevention and Genetics, Istituto Europeo di Oncologia (IEO), Milan, Italy ${ }^{78}$ Division of Experimental Oncology, Centro di Riferimento Oncologico (CRO), IRCCS, Aviano (PN), Italy ${ }^{79}$ Hospital Clinico San Carlos, Madrid, Spain ${ }^{80}$ Department of Obstetrics/Gynocology, Medical University of Vienna, Vienna, Austria ${ }^{81}$ Clinical Genetics Branch, Division of Cancer Epidemiology and Genetics, National Cancer Institute, Bethesda, Maryland, USA 82 Medical Genetics, Mayo Clinic Rochester, Rochester, Minnesota, USA ${ }^{83}$ Department of Clinical Biochemistry, Copenhagen University Hospital, Copenhagen, Denmark ${ }^{84}$ Hereditary Cancer Program, Catalan Institute of Oncology, Gran Via de l'Hospitalet, Barcelona, Spain ${ }^{85}$ Dana-Farber Cancer Institute, Boston, Massachusetts, USA ${ }^{86}$ Department of Gynaecological Oncology, University College London, Elizabeth Garrett Anderson (EGA) Institute for Women's Health, London, UK ${ }^{87}$ Women's College Research Institute, Toronto, Ontario, Canada ${ }^{88}$ Department of Epidemiology and Genetics, $\mathrm{H}$. Lee Moffitt Cancer Center, Tampa, Florida, USA ${ }^{89}$ MOD SQUAD, Modifier Study of Quantitative Effects on Disease ${ }^{90}$ Human Cancer Genetics Programme, Spanish National Cancer Research Centre, Madrid, Spain ${ }^{91}$ Helsinki University Central Hospital, Department of Obstetrics and Gynecology, Helsinki, Finland ${ }^{92}$ Division of Surgical, Molecular and Ultrastructural Pathology, Department of Oncology, University of Pisa, Pisa University Hospital, Pisa, Italy ${ }^{93}$ Department of Medicine, University of California, San Francisco, San Francisco, California, USA ${ }^{94}$ Department of Epidemiology and Biostatistics, University of California, San Francisco, San Francisco, California, USA ${ }^{95}$ Cancer Risk Program, Helen Diller Family Cancer Center, University of California, San Francisco, San Francisco, California, USA ${ }^{96}$ German Cancer Research Center (DKFZ), Heidelberg, Germany ${ }^{97}$ Istituto Oncologico Veneto-IRCCS, Immunology and Molecular Oncology Unit, Padua, Italy ${ }^{98}$ Department of Population Sciences, The Beckman Research Institute of the City of Hope, Duarte, California, USA ${ }^{99}$ Women's Cancer Research Institute at the Samuel Oschin Cancer Institute, Division of Gynecologic Oncology, Cedars-Sinai Medical Center, Los Angeles, California, USA ${ }^{100}$ Department of Obstetrics and Gynecology, David Geffen School of Medicine at the University of California Los Angeles, Los Angeles, California, USA ${ }^{101}$ Beth Israel Deaconess Medical Center, Boston, Massachusetts, USA ${ }^{102}$ Comprehensive Cancer Center, Department of Molecular Virology, Immunology and Medical Genetics, Division of Human Genetics, Department of Internal Medicine, Ohio State University, Columbus, Ohio, USA 103 Division of Clinical Cancer Genetics, City of Hope Cancer Center, Duarte, California, USA ${ }^{104}$ University of Chicago Medical Center, Chicago, Illinois, USA ${ }^{105}$ Cancer Genomics Laboratory, Centre Hospitalier Universitaire de Quebec, Québec City, Quebec, Canada ${ }^{106}$ Université Laval, Centre de recherche du Centre Hospitalier Universitaire de Quebéc (CHUQ), Québec City, Quebec, Canada ${ }^{107}$ Northshore University Health System, Evanston, Illinois, USA 108 Department of Pathology, Landspitali University Hospital, Reykjavik, Iceland ${ }^{109}$ QIMR GWAS Collective, Queensland Institute of Medical Research, Brisbane, Australia ${ }^{110}$ Clalit Health Services National Cancer Control Center, Department of Community Medicine and Epidemiology, Carmel Medical Center and B. Rappaport Faculty of Medicine, Technion, Haifa, Israel ${ }^{111}$ Division of Cancer Epidemiology, German Cancer Research Center, Heidelberg, Germany ${ }^{112}$ Institute for Medical Biometrics and Epidemiology, University Clinic Hamburg-Eppendorf, Hamburg, Germany ${ }^{113}$ Dr. Margarete Fischer-Bosch-Institute of Clinical Pharmacology, Stuttgart, Germany ${ }^{114}$ University Tübingen, Tübingen, Germany ${ }^{115}$ GENICA, Gene Environment Interaction and Breast Cancer in Germany ${ }^{116}$ Melbourne Collaborative Cohort Study (MCCS), Cancer Epidemiology Centre, The Cancer Council Victoria, Melbourne, Australia ${ }^{117}$ Institute for Cancer Studies, Department of Oncology, Faculty of Medicine, Dentistry and Health, University of Sheffield, Sheffield, UK ${ }^{118}$ Academic Unit of Pathology, Department of Neuroscience, Faculty of Medicine, 
Dentistry and Health, University of Sheffield, Sheffield, UK ${ }^{119}$ Department of Cancer Biology, Dana-Farber Cancer Institute, Boston, Massachusetts, USA ${ }^{120}$ Molecular Diagnostics Laboratory Institute of Radioisotopes and Radiodiagnostic Products, National Centre for Scientific Research 'Demokritos', Athens, Greece ${ }^{121}$ Department of Medical Oncology, Papageorgiou Hospital, Aristotle University of Thessaloniki School of Medicine, Thessaloniki, Greece ${ }^{122}$ Hellenic Cooperative Oncology Group, Greece ${ }^{123}$ University of California at Los Angeles, David Geffen School of Medicine, Department of Medicine, Division of Hematology and Oncology, Los Angeles, California, USA ${ }^{124}$ University Hospital Erlangen, Department of Gynecology and Obstetrics, Erlangen, Germany ${ }^{125}$ Cancer Research UK Epidemiology and Genetics Group, Department of Epidemiology and Population Health, London School of Hygiene and Tropical Medicine, London, UK ${ }^{126}$ Vesalius Research Center (VRC), VIB, Leuven, Belgium ${ }^{127}$ Multidisciplinary Breast Center, University Hospitals Leuven, Leuven, Belgium ${ }^{128}$ Institute of Pathology, Städtisches Klinikum Karlsruhe, Karlsruhe, Germany ${ }^{129}$ Center for Primary Health Care Research, University of Lund, Lund, Sweden ${ }^{130}$ Laboratory of Cancer Genetics, Department of Clinical Genetics and Biocenter Oulu, University of Oulu, Oulu University Hospital, Oulu, Finland ${ }^{131}$ Department of Pharmacology, Mayo Clinic, Rochester, Minnesota, USA ${ }^{132}$ Center for Applied Genomics, Children's Hospital of Philadelphia, Philadelphia, Pennsylvania, USA

\section{Abstract}

Germline BRCAl mutations predispose to breast cancer. To identify genetic modifiers of this risk, we performed a genome-wide association study in 1,193 individuals with BRCAl mutations who were diagnosed with invasive breast cancer under age 40 and 1,190 BRCAl carriers without breast cancer diagnosis over age 35. We took forward 96 SNPs for replication in another 5,986 BRCA1 carriers (2,974 individuals with breast cancer and 3,012 unaffected individuals). Five SNPs on 19 p13 were associated with breast cancer risk $\left(P_{\text {trend }}=2.3 \times 10^{-9}\right.$ to $\left.P_{\text {trend }}=3.9 \times 10^{-7}\right)$, two of which showed independent associations (rs8170, hazard ratio $(\mathrm{HR})=1.26,95 \% \mathrm{CI} 1.17-1.35$; rs $2363956 \mathrm{HR}=0.84,95 \%$ CI 0.80-0.89). Genotyping these SNPs in 6,800 population-based breast cancer cases and 6,613 controls identified a similar association with estrogen receptornegative breast cancer (rs2363956 per-allele odds ratio $(\mathrm{OR})=0.83,95 \% \mathrm{CI} 0.75-0.92, P_{\text {trend }}=$ $0.0003)$ and an association with estrogen receptor-positive disease in the opposite direction $(\mathrm{OR}=$ $1.07,95 \%$ CI 1.01-1.14, $P_{\text {trend }}=0.016$ ). The five SNPs were also associated with triple-negative breast cancer in a separate study of 2,301 triple-negative cases and 3,949 controls $\left(P_{\text {trend }}=1 \times\right.$ $10^{-7}$ to $P_{\text {trend }}=8 \times 10^{-5} ;$ rs 2363956 per-allele $\mathrm{OR}=0.80,95 \%$ CI $0.74-0.87, P_{\text {trend }}=1.1 \times$ $\left.10^{-7}\right)$.

Pathogenic BRCA1 and BRCA2 mutations confer high risks of breast and ovarian cancer. Variation in risk estimates by degree of family history suggests that these risks are modified by other genetic variants ${ }^{1-5}$. Recent studies from the Consortium of Investigators of Modifiers of BRCA1/2 (CIMBA) have demonstrated that common breast cancer susceptibility alleles, identified through genome-wide association studies (GWAS) in the general population ${ }^{6-9}$, are also associated with the risk of developing breast cancer in $B R C A 1$ or $B R C A 2$ mutation carriers ${ }^{10,11}$. However, although five of six alleles were associated with risk of breast cancer for $B R C A 2$ mutation carriers, only two polymorphisms (in the TOX3 and 2q35 regions) were associated with risk for $B R C A 1$ carriers. These findings are consistent with the distinct pathology of breast cancer in BRCAl tumors ${ }^{12,13}$ and suggest that the genetic variants that modify breast cancer risk for BRCAl mutation carriers may differ from the modifiers of risk for $B R C A 2$ carriers or for non-carriers.

To search for genetic loci associated with breast cancer in $B R C A l$ carriers, we conducted a two-stage GWAS. In stage 1, we genotyped 2,500 BRCAl carriers using the Illumina 
Infinium 610K array, which included 620,901 SNPs. Mutation carriers were selected on the basis of an invasive breast cancer diagnosis at under 40 years of age $(n=1,250)$ or the absence of breast cancer when 35 years of age or older $(n=1,250)$. After quality control exclusions, 2,383 carriers (1,193 unaffected and 1,190 affected) from 20 centers in 11 different countries and 555,616 SNPs were available for analysis (Supplementary Tables 1 and 2). Genotype associations were evaluated using a 1 degree-of-freedom (d.f.) score test for trend, based on modeling the retrospective likelihood of the observed genotypes conditional on the disease phenotypes, stratified by country of residence. A kinship-adjusted version of the score test statistic was used to allow for the dependence between related individuals.

There was little evidence for inflation in the test statistic of association (inflation factor $(\lambda)=$ 1.036; Supplementary Fig. 1). Ninety-six SNPs were significant at the $P<10^{-4}$ level compared with 55.6 SNPs which were expected by chance. In stage 2, we genotyped 86 of these SNPs, seven surrogate SNPs (within $10 \mathrm{~kb}$ of the significant SNPs and pair-wise $r^{2}>$ 0.90 ) and three additional SNPs in 6,332 BRCAl carriers. After quality control exclusions, 89 SNPs and 5,986 BRCAl mutation carriers (3,012 unaffected and 2,974 affected) were used in the stage 2 analysis. The most significant associations were for five SNPs on 19p13 $(P<0.002)$, which had hazard ratios in the same direction as in stage 1 (Table 1 and Supplementary Table 3$)$. In the combined analysis of stage 1 and 2 , there was strong evidence of association ${ }^{14}$ with breast cancer for these SNPs $\left(P=2.3 \times 10^{-9}\right.$ to $P=3.9 \times$ $\left.10^{-7}\right)$.

The minor alleles of rs 8170 and rs 4808611 were associated with an increased breast cancer risk for $B R C A 1$ carriers (per allele HR $=1.26,95 \%$ CI $1.17-1.35$ for both SNPs). In contrast, SNPs rs8100241, rs 2363956 and rs 3745185 were associated with decreased breast cancer risk ( $\mathrm{HR}=0.84,95 \% \mathrm{CI} 0.80-0.89$ for rs 8100241 and rs $2363956 ; \mathrm{HR}=0.86,95 \%$ CI 0.81-0.91 for rs3745185) (Table 1). The HR estimates for rs 8170 and rs4808611 were similar in stages 1 and 2, but for rs8100241, rs2363956 and rs3745185, the HRs were stronger in stage 1; this may be due to the sample selection criteria for stage 1 or a 'winner's curse' effect ${ }^{15}$. There was no evidence of heterogeneity in the HR estimates among the countries of residence in stages 1 and 2 combined (Fig. 1 ; rs8170, $P=0.10$; rs $4808611, P=$ $0.14 ;$ rs8100241, $P=0.18 ;$ rs2363956, $P=0.17$; and rs3745185, $P=0.48$ ).

The strength of the association with breast cancer could also be affected by the inclusion of prevalent cases if these SNPs were associated with breast cancer survival. To address this possibility, we excluded breast cancer cases diagnosed with the disease $>5$ years before study entry. The HR estimates were similar to the overall analysis after this exclusion (Supplementary Table 4). This indicates that the inclusion of prevalent breast cancer cases was unlikely to have influenced the overall results.

To investigate whether any of these SNPs were associated with ovarian cancer risk for $B R C A l$ carriers, we analyzed the data within a competing risks framework and estimated HR simultaneously for breast and ovarian cancer. There was no evidence of association with ovarian cancer risk for any of the SNPs, and the breast cancer associations were virtually identical to the primary analysis both in terms of significance and in the HR estimates (Table 2). We repeated the breast cancer association analysis after excluding all individuals who developed ovarian cancer either before or after a breast cancer diagnosis. Despite the sample size reduction, the top four SNPs remained significant at $P<10^{-7}$ and the HR estimates were identical to the analysis which included individuals with ovarian cancer as unaffected individuals (Supplementary Table 4). We also evaluated ovarian cancer associations after excluding individuals with ovarian cancer who were recruited $>3$ years after their cancer diagnosis in order to account for a potential survival bias. No significant associations were 
observed after this exclusion ( $P_{\text {trend }}=0.44$ to $P_{\text {trend }}=0.96$ using competing risk analysis). We conclude that the associations with breast cancer were not confounded by the competing risk of ovarian cancer.

We evaluated the SNP associations by the predicted functional consequences of BRCAI mutation type ${ }^{16-18}$. Class 1 mutations correspond to loss-of-function mutations and are expected to result in a reduced transcript or protein level due to nonsense-mediated RNA decay, whereas class 2 mutations are likely to generate stable proteins with potential residual or dominant negative function ${ }^{18-20}$. Among class 1 mutation carriers (combined stage 1 and $2, n=5,732)$, the five most significant associated SNPs included rs6994019, an intronic SNP in $M M P 16$ on chromosome $8\left(P_{\text {trend }}=2.9 \times 10^{-6}\right)$ and four SNPs in the 19 p13 region $\left(P_{\text {trend }}=7.6 \times 10^{-6}\right.$ to $\left.P_{\text {trend }}=1.6 \times 10^{-4}\right)$. The MMP16 SNP rs6994019 was the ninth most significant SNP in the primary analysis of all mutations combined $\left(P_{\text {trend }}=2.7 \times 10^{-4}\right.$ in stage 1 and 2 combined; Supplementary Table 3). The strongest association with breast cancer risk for carriers of class 2 mutations was at the five SNPs in the $19 \mathrm{p} 13$ region $\left(P_{\text {trend }}\right.$ $=1.8 \times 10^{-6}$ to $P_{\text {trend }}=1.2 \times 10^{-4}$; Supplementary Table 3 ). The HR estimates for the five SNPs in 19p13 were larger for class 2 mutations, but the differences between class 1 and class 2 mutations were significant for only rs8170 and rs3745185 ( $P=0.03$ and $P=0.004$, respectively). These differences might reflect a stronger modifying effect on breast cancer risk for tumors retaining residual or dominant negative BRCA1 function.

Tumor estrogen or progesterone receptor status was available for 1,197 breast cancer cases in stage 1 and 2 combined. A case-only analysis revealed significant differences in the associations for the 19p13 SNPs between estrogen receptor-positive and estrogen receptornegative disease and between estrogen receptor- or progesterone receptor-positive and estrogen receptor- and progesterone receptor-negative disease, particularly for SNPs rs8100241, rs2363956 and rs3745185 ( $P=0.002$ to $P=0.04$; Supplementary Table 5). The OR estimates suggest that these SNPs are more strongly associated with estrogen receptornegative disease.

The two most significant SNPs (rs8170 and rs4808611) were strongly correlated $\left(r^{2}=0.87\right)$ in the BRCA1 samples but displayed a low correlation with the other associated SNPs $\left(r^{2}<\right.$ $0.23)$. rs 8100241 and $\mathrm{rs} 2363956$ were perfectly correlated $\left(r^{2}=1\right)$, whereas the least significant SNP, rs3745185, had weaker correlations with both sets of SNPs $\left(r^{2}=0.17\right.$ and $r^{2}=0.74$ with rs8170 and rs8100241, respectively).

To evaluate the contribution of the 19p13 locus to breast cancer risk in the general population, we genotyped rs8170 and rs2363956 in 6,800 breast cancer cases and 6,613 controls from the SEARCH (Studies of Epidemiology and Risk Factors in Cancer Heredity) study in the UK. Neither SNP was associated with overall breast cancer risk $(P=0.65$ and $P$ $=0.79$; Table 3). However, stratification of tumors by estrogen receptor status indicated that both SNPs were associated with estrogen receptor-negative breast cancer (rs8170, per-allele $\mathrm{OR}=1.21,95 \% \mathrm{CI} 1.07-1.37, P=0.0029$ and $\mathrm{rs} 2363956, \mathrm{OR}=0.83,95 \% \mathrm{CI} 0.75-0.92, P$ $=0.0003$; Table 3$)$. These effect sizes were similar to the estimated HRs for BRCAl carriers, consistent with the observation that $B R C A 1$ mutations predispose predominately to estrogen receptor-negative disease. Weaker associations were observed in the opposite direction for estrogen receptor-positive disease (rs8170, per-allele $\mathrm{OR}=0.91,95 \% \mathrm{CI} 0.84-0.98, P=$ 0.011 and rs $2363956, \mathrm{OR}=1.07,95 \%$ CI 1.01-1.14, $P=0.016$ ). Similar patterns were observed when tumors were stratified by progesterone receptor status or estrogen receptor and progesterone receptor status combined (Table 3).

The majority of breast tumors in BRCAl carriers exhibit a triple-negative (estrogen receptor, progesterone receptor and HER2 negative) phenotype. To evaluate the association of the 
19p13 locus with triple-negative disease in the general population, we obtained genotype data for the five SNPs from up to 2,301 cases from 15 centers in six countries involved in the triple-negative breast cancer consortium (TNBCC). Genotype data from up to 3,949 geographically matched controls were also available (Supplementary Table 5). All SNPs were associated with triple-negative breast cancer, and the ORs were comparable to the HRs seen in the BRCAl carriers and the ORs for estrogen receptor-negative breast cancer seen in the SEARCH population-based study (rs 2363956 , per-allele $\mathrm{OR}=0.80,95 \%$ CI $0.74-0.87$, $P=1.1 \times 10^{-7}$ and rs8170, OR $=1.28,95 \%$ CI 1.16-1.41, $P=1.2 \times 10^{-6}$; Table 3 and Supplementary Table 5).

Two of the SNPs (rs8170 and rs2366956) were genotyped in 2,486 BRCA2 mutation carriers as part of an ongoing GWAS. Neither SNP was associated with breast cancer risk for $B R C A 2$ carriers $\left(P_{\text {trend }}=0.17\right.$ and $\left.P_{\text {trend }}=0.07\right)$, but the HR estimates were in line with the ORs estimated for estrogen receptor-positive disease in the SEARCH study (Table 3).

All five SNPs were located in a region that spans $39 \mathrm{~kb}$ on 19p13 (Fig. 2). In an analysis for the joint effect of these SNPs on breast cancer risk for BRCAl carriers, it was not possible to distinguish between rs8170 and rs4808611, as neither SNP improved the model fit significantly when the other was included $(P=0.11$ and $P=0.22$ for rs 8170 and rs4808611, respectively). rs8100241 was retained in preference to $\mathrm{rs} 3745185$ ( $P$ for inclusion of rs3745185 in model $=0.79$ ). Thus, the most parsimonious model included SNPs rs8170 and either rs8100241 or rs2363956 ( $P$ for inclusion $=7.7 \times 10^{-5}$ and $P=6.7 \times 10^{-5}$ for rs 8170 and rs8100241, respectively) and had a 2 d.f. $P=6.3 \times 10^{-13}$ for inclusion of both SNPs. This suggests that these associations may be driven by a single causative variant that is partially correlated with all five SNPs. To investigate this further, we evaluated the associations for SNPs identified through the 1000 Genomes Project using imputation. 1,055 SNPs in a $300-\mathrm{kb}$ interval with a minor allele frequency $>0.01$ in samples of European ancestry, were evaluated. Thirty-one SNPs, none of which were genotyped in stage 1, displayed $P<1.76 \times 10^{-9}$ (Fig. 2 and Supplementary Table 3). The most significant associations with the imputed genotypes in stage 1 and 2 combined were for eight perfectly correlated SNPs within a 13-kb region (the most significant SNP was rs4808075, $P=9.4 \times$ $10^{-12}$; Supplementary Table 3). These SNPs were correlated with the four genotyped SNPs $\left(r^{2}=0.37\right.$ to $r^{2}=0.58$ based on the 1000 Genomes Project data; Supplementary Fig. 2). This suggests that one or more of these imputed SNPs may be causally associated with breast cancer risk. However, some rare SNPs may have been missed because the 1000 Genome Project data used were based on the resequencing of only 56 individuals. Therefore, the possibility that the association is driven by a rarer variant, or a more cryptic common variant not detected in the resequencing, cannot yet be ruled out.

Of the five genotyped SNPs in the region and the eight most significant imputed SNPs, only rs 8170 and rs 2363956 were located in coding regions. The smaller 13-kb region, defined by the most strongly associated SNPs, contains three genes: ABHD8 (encoding abhydrolase domain containing 8), ANKLE1 (encoding ankyrin repeat and LEM domain containing 1) and C19orf62. The eight most significant imputed SNPs were clustered in and around ANKLE1, which encodes a protein of undefined function. However, C19orf62, which encodes MERIT40 (Mediator of Rap80 Interactions and Targeting $40 \mathrm{kD}$ ), is a more plausible genetic modifier of breast cancer in BRCAl carriers because MERIT40 interacts with BRCA1 in a protein complex. MERIT40 is a component of the BRCA1 A complex containing BRCA1-BARD1, Abraxas1, RAP80, BRCC36 and BRCC45 that is required for recruitment and retention of the BRCA1-BARD1 ubiquitin ligase and the BRCC36 deubiquitination enzyme at sites of DNA damage ${ }^{21-23}$. Thus, a variant that modifies MERIT40 function or expression might influence BRCA1-dependent DNA repair and checkpoint activity in mammary epithelial cells of BRCAl carriers sufficiently, before loss 
of the wildtype BRCA1 allele, to increase the risk of breast cancer. However, until the SNPs that increase risk of cancer have been definitively linked to MERIT40, it remains possible that the other genes in the region or genes influenced by long range chromatin remodeling or by transcriptional events account for the breast cancer association.

Genetic variation at this locus, in combination with other risk modifiers, may prove useful in individual cancer risk assessment for breast cancer in BRCAl carriers. In addition, understanding the functional basis of this association may provide important insights into the etiology of $B R C A 1$-associated breast cancer and hormone receptor-negative breast cancer in the general population. Our results suggest that GWAS in BRCAl mutation carriers or GWAS restricted to specific breast cancer subtypes may identify further breast cancer susceptibility variants.

\section{METHODS}

Methods and any associated references are available in the online version of the paper at http://www.nature.com/naturegenetics/.

\section{URLs}

1000 Genomes Project, http://www.1000genomes.org; MACH software, http://www.sph.umich.edu/csg/yli/mach/index.html/.

\section{Supplementary Material}

Refer to Web version on PubMed Central for supplementary material.

\section{Acknowledgments}

Financial support for this study was provided by the Breast Cancer Research Foundation (BCRF), Susan G. Komen for the Cure and US National Institutes of Health grant CA128978 to F.J.C. and by Cancer Research UK to D.F.E. and A.C.A. A.C.A. is a Cancer Research UK Senior Cancer Research Fellow and D.F.E. is a Cancer Research UK Principal Research Fellow. The authors thank Cancer Genetic Markers of Susceptability (CGEMS) and Wellcome Trust Case Control Consortium (WTCCC) for provision of genotype data from controls. Study specific acknowledgments listed in Supplementary Note.

\section{References}

1. Antoniou A, et al. Average risks of breast and ovarian cancer associated with $B R C A 1$ or $B R C A 2$ mutations detected in case series unselected for family history: a combined analysis of 22 studies. Am J Hum Genet. 2003; 72:1117-1130. [PubMed: 12677558]

2. Antoniou AC, et al. The BOADICEA model of genetic susceptibility to breast and ovarian cancers: updates and extensions. Br J Cancer. 2008; 98:1457-1466. [PubMed: 18349832]

3. Begg CB, et al. Variation of breast cancer risk among BRCA1/2 carriers. J Am Med Assoc. 2008; 299:194-201.

4. Milne RL, et al. The average cumulative risks of breast and ovarian cancer for carriers of mutations in BRCA1 and BRCA2 attending genetic counseling units in Spain. Clin Cancer Res. 2008; 14:2861-2869. [PubMed: 18451254]

5. Simchoni S, et al. Familial clustering of site-specific cancer risks associated with BRCA1 and BRCA2 mutations in the Ashkenazi Jewish population. Proc Natl Acad Sci USA. 2006; 103:37703774. [PubMed: 16537453]

6. Easton DF, et al. Genome-wide association study identifies novel breast cancer susceptibility loci. Nature. 2007; 447:1087-1093. [PubMed: 17529967]

7. Hunter DJ, et al. A genome-wide association study identifies alleles in FGFR2 associated with risk of sporadic postmenopausal breast cancer. Nat Genet. 2007; 39:870-874. [PubMed: 17529973] 
8. Stacey SN, et al. Common variants on chromosomes $2 \mathrm{q} 35$ and $16 \mathrm{q} 12$ confer susceptibility to estrogen receptor-positive breast cancer. Nat Genet. 2007; 39:865-869. [PubMed: 17529974]

9. Stacey SN, et al. Common variants on chromosome $5 \mathrm{p} 12$ confer susceptibility to estrogen receptorpositive breast cancer. Nat Genet. 2008; 40:703-706. [PubMed: 18438407]

10. Antoniou AC, et al. Common breast cancer-predisposition alleles are associated with breast cancer risk in BRCA1 and BRCA2 mutation carriers. Am J Hum Genet. 2008; 82:937-948. [PubMed: 18355772]

11. Antoniou AC, et al. Common variants in LSP1, 2q35 and 8q24 and breast cancer risk for BRCA1 and BRCA2 mutation carriers. Hum Mol Genet. 2009; 18:4442-4456. [PubMed: 19656774]

12. Lakhani SR, et al. The pathology of familial breast cancer: predictive value of immunohistochemical markers estrogen receptor, progesterone receptor, HER-2, and p53 in patients with mutations in BRCA1 and BRCA2. J Clin Oncol. 2002; 20:2310-2318. [PubMed: 11981002]

13. Lakhani SR, et al. Prediction of $B R C A 1$ status in patients with breast cancer using estrogen receptor and basal phenotype. Clin Cancer Res. 2005; 11:5175-5180. [PubMed: 16033833]

14. Wellcome Trust Case Control Consortium. Genome-wide association study of 14,000 cases of seven common diseases and 3,000 shared controls. Nature. 2007; 447:661-678. [PubMed: 17554300]

15. Zollner S, Pritchard JK. Overcoming the winner's curse: estimating penetrance parameters from case-control data. Am J Hum Genet. 2007; 80:605-615. [PubMed: 17357068]

16. Buisson M, Anczukow O, Zetoune AB, Ware MD, Mazoyer S. The 185delAG mutation (c. 68_69delAG) in the BRCA1 gene triggers translation reinitiation at a downstream AUG codon. Hum Mutat. 2006; 27:1024-1029. [PubMed: 16941470]

17. Mazoyer S, et al. A BRCA1 nonsense mutation causes exon skipping. Am J Hum Genet. 1998; 62:713-715. [PubMed: 9497265]

18. Perrin-Vidoz L, Sinilnikova OM, Stoppa-Lyonnet D, Lenoir GM, Mazoyer S. The nonsensemediated mRNA decay pathway triggers degradation of most BRCA1 mRNAs bearing premature termination codons. Hum Mol Genet. 2002; 11:2805-2814. [PubMed: 12393792]

19. Antoniou AC, et al. RAD51 $135 \mathrm{G} \rightarrow \mathrm{C}$ modifies breast cancer risk among BRCA2 mutation carriers: results from a combined analysis of 19 studies. Am J Hum Genet. 2007; 81:1186-1200. [PubMed: 17999359]

20. Liu HX, Cartegni L, Zhang MQ, Krainer AR. A mechanism for exon skipping caused by nonsense or missense mutations in BRCA1 and other genes. Nat Genet. 2001; 27:55-58. [PubMed: 11137998]

21. Feng L, Huang J, Chen J. MERIT40 facilitates BRCA1 localization and DNA damage repair. Genes Dev. 2009; 23:719-728. [PubMed: 19261748]

22. Shao G, et al. MERIT40 controls BRCA1-Rap80 complex integrity and recruitment to DNA double-strand breaks. Genes Dev. 2009; 23:740-754. [PubMed: 19261746]

23. Wang B, Hurov K, Hofmann K, Elledge SJ. NBA1, a new player in the Brca1 A complex, is required for DNA damage resistance and checkpoint control. Genes Dev. 2009; 23:729-739. [PubMed: 19261749] 

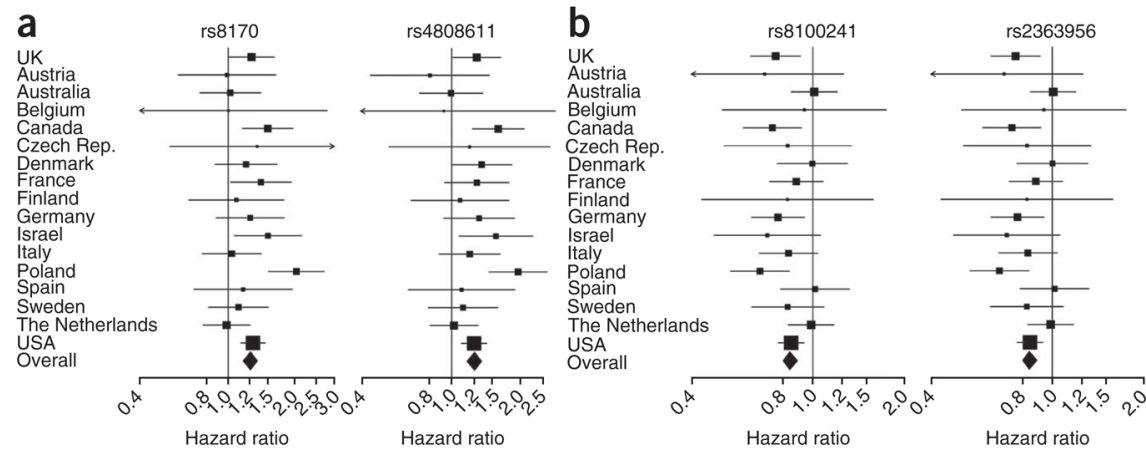

Figure 1.

Forest plots of the associations by country of residence of $B R C A 1$ mutation carriers in the combined stage 1 and stage 2 samples. (a,b) Squares indicate the country specific per-allele HR estimates for SNPs rs8170, rs4808611 (a) and rs8100241, rs2363956 (b). The area of the square is proportional to the inverse of the variance of the estimate. Horizontal lines indicate $95 \%$ CIs. 

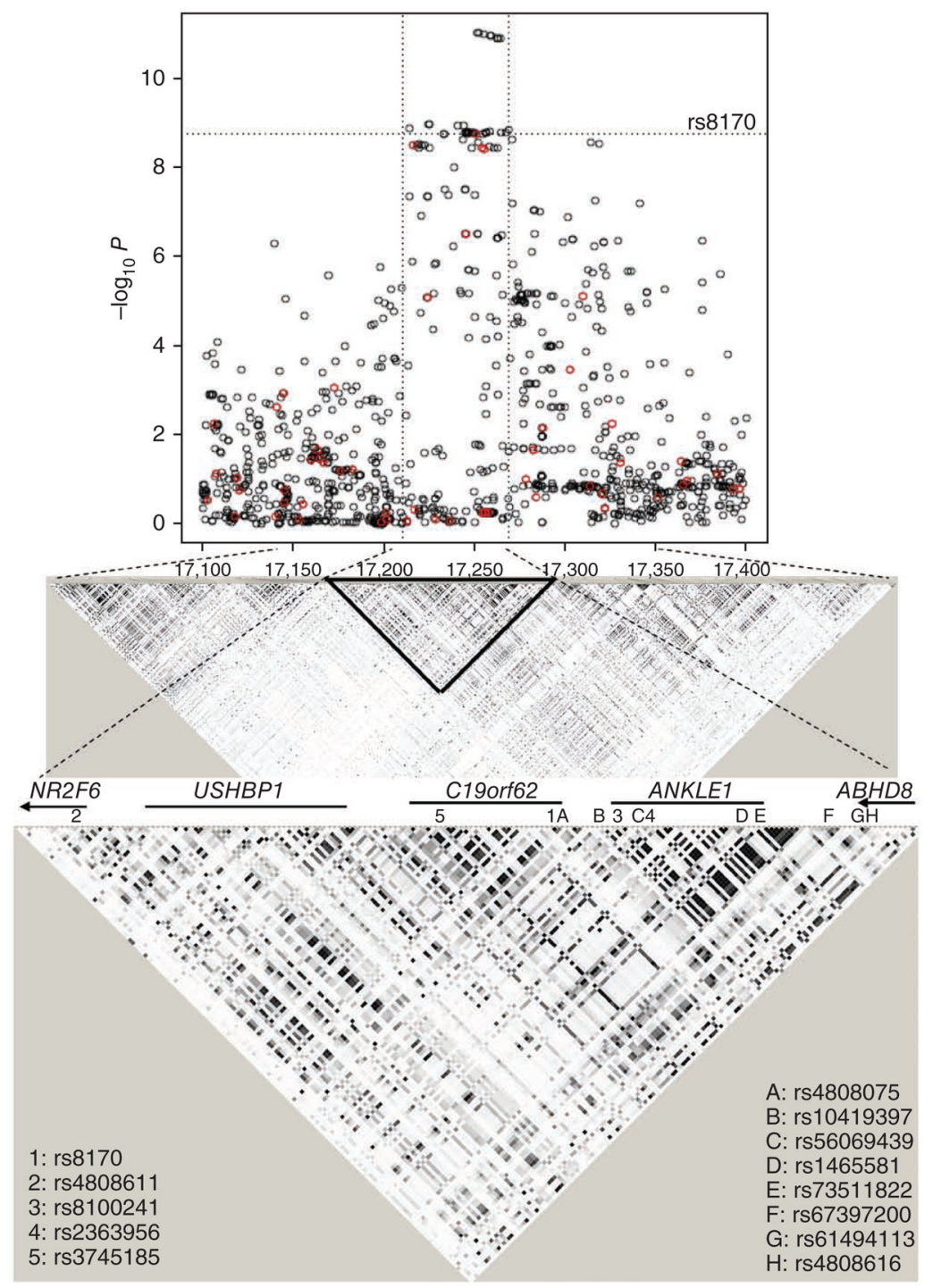

Figure 2.

Above, results of the kinship-adjusted score test statistic (1 d.f.) by position (kb) in stage 1 and 2 samples combined for genotyped and imputed SNPs in the associated region (chromosome 19, positions 17,100-17,400 kb). Genotyped SNPs in stages 1 or 2 are shown in red and imputed SNPs are shown in black. The horizontal dotted line indicates the $P$ values for the strongest association among genotyped SNPs (rs8170). At middle, the linkage disequilibrium (LD) blocks around the top five associated SNPs (chromosome 19, positions $17,150-17,350 \mathrm{~kb}$ ) in the combined analysis of stage 1 and stage 2 samples based on the 1000 Genomes Project data for the samples of European ancestry. Squares in the LD blocks indicate pairwise correlations between the SNPs $\left(r^{2}\right)$ by grayscale (darker symbols indicate correlations closer to 1). Below, details of the region containing the most significantly associated genotyped and imputed SNPs (chromosome 19, positions 17,210-17,268 kb). Location of genotyped SNPs shown by numbers $1-5$ and the eight most significantly associated imputed SNPs are shown in letters A-H $\left(P=9.0 \times 10^{-12}\right.$ to $\left.P=1.0 \times 10^{-11}\right)$. 


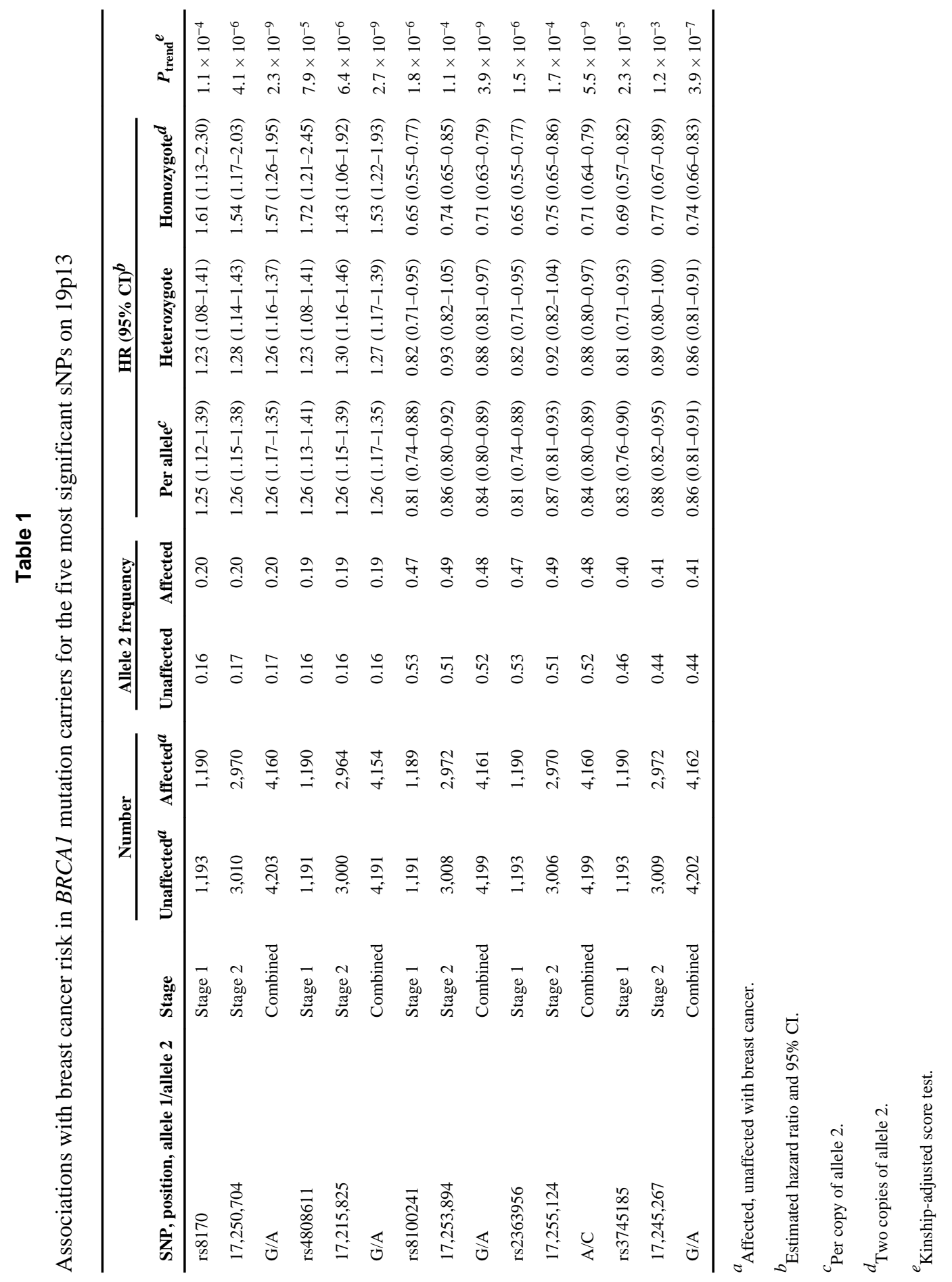

Nat Genet. Author manuscript; available in PMC 2011 October 1. 


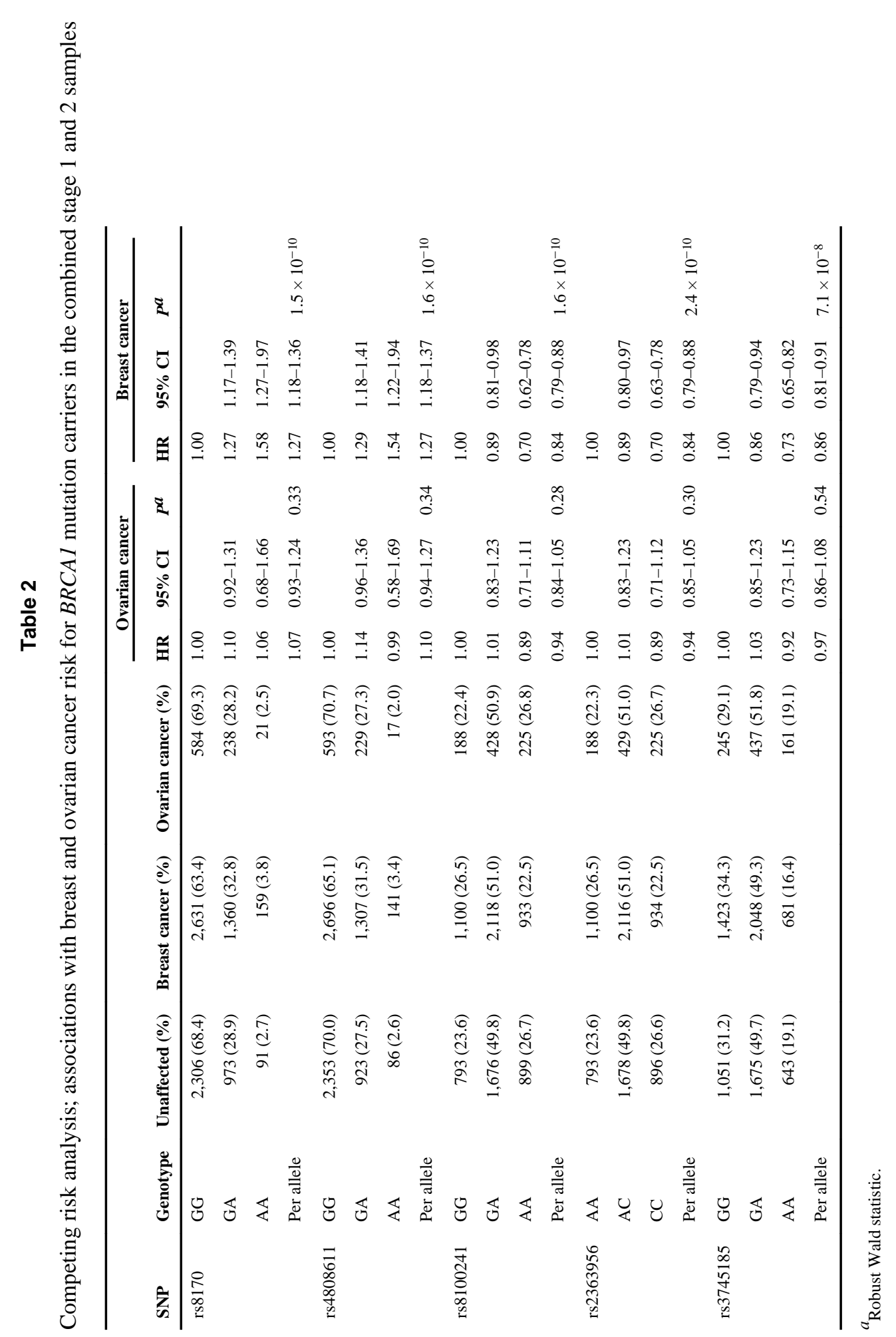




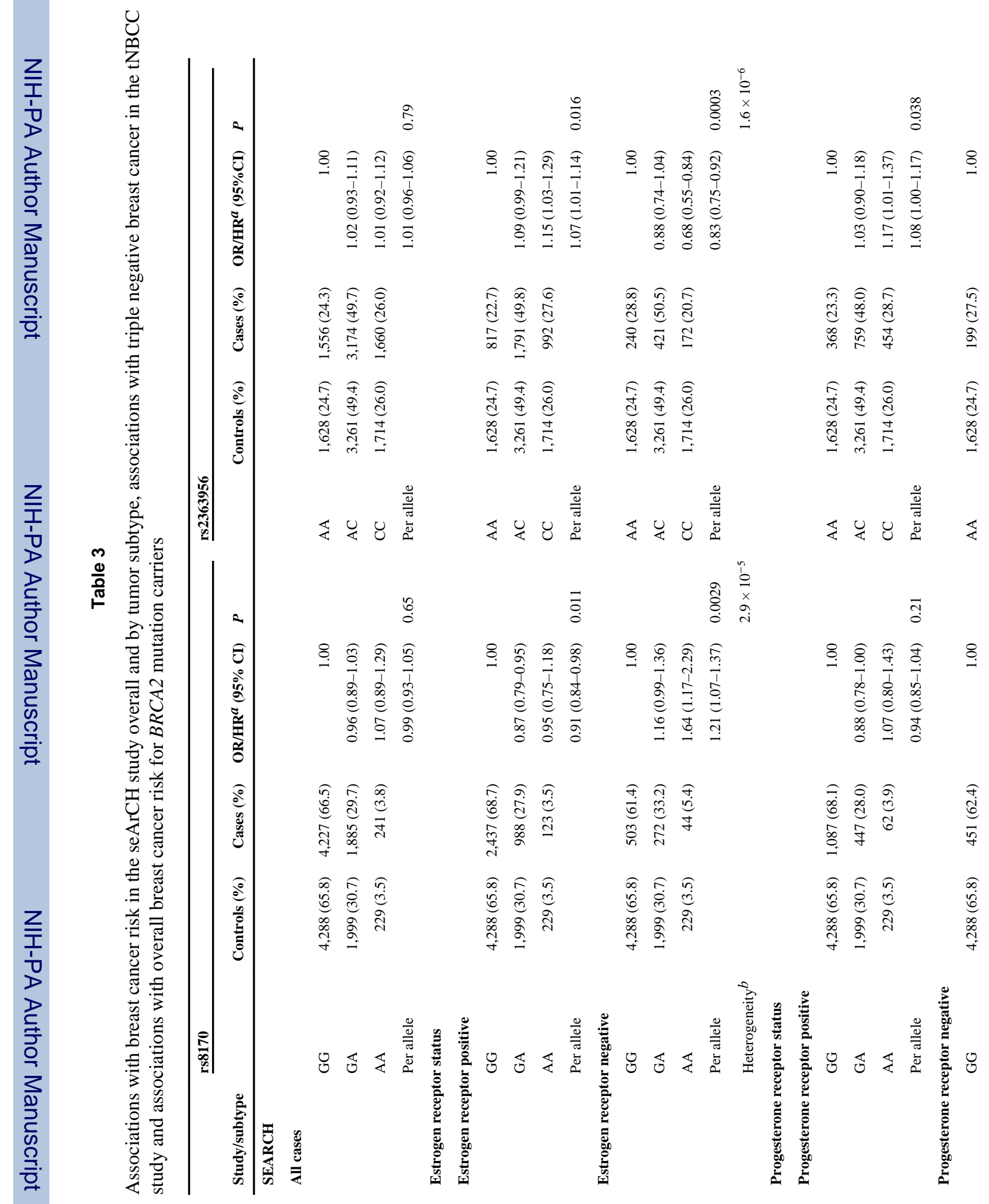


Antoniou et al.

Page 16

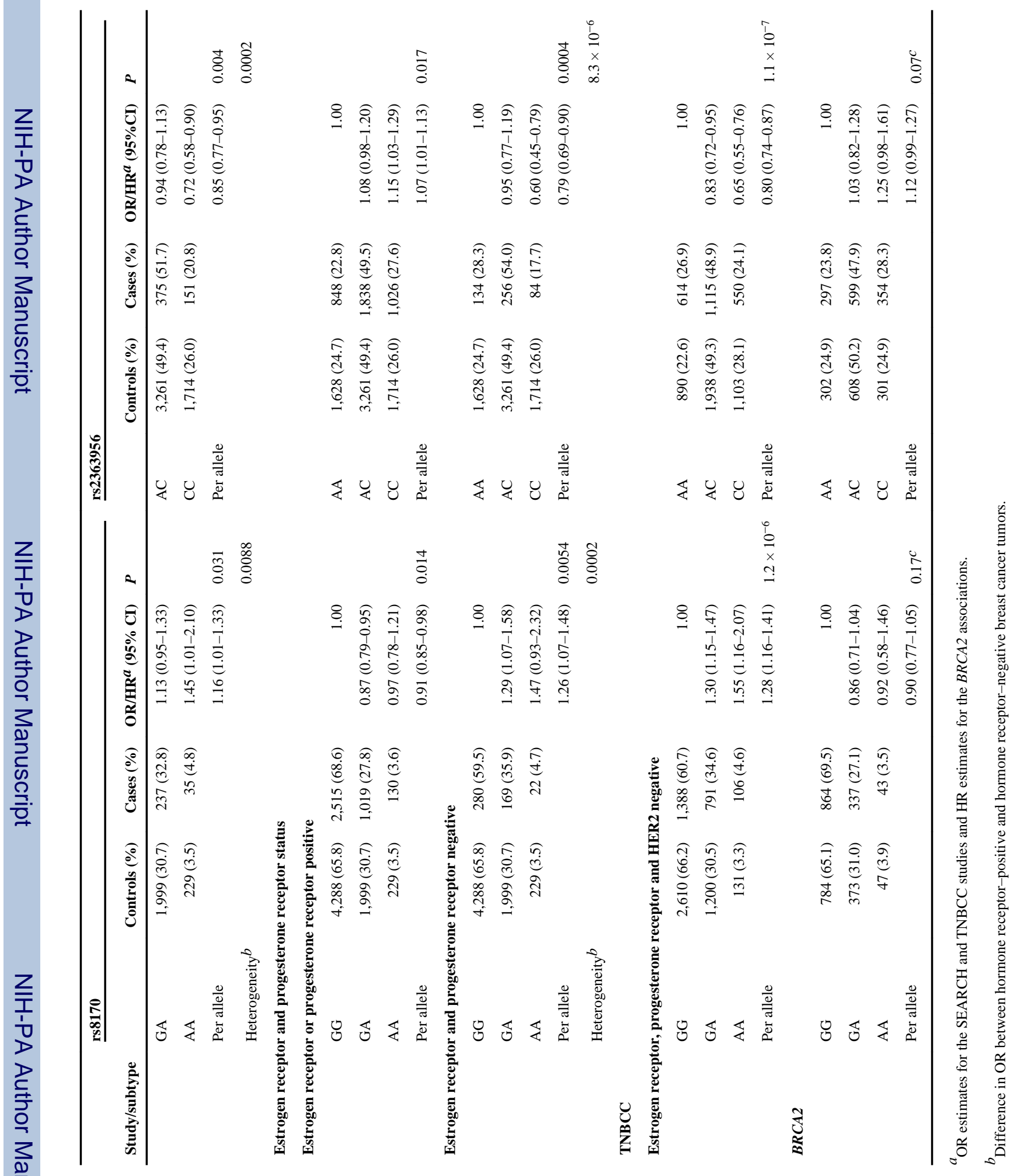

Nat Genet. Author manuscript; available in PMC 2011 October 1. 
